\title{
Concepciones de tecnología en docentes universitarios de ciencias
}

\author{
Conceptions of Technology in University Teachers of Sciences
}

\section{Concepções de tecnología em estudantes universitários educacionais de ciências}

\author{
Néstor Cardoso-Erlam \\ Edna Eliana Morales-Oliveros ${ }^{2}$
}

Recibido: mayo de 2017

Aceptado: agosto de 2017

Para citar este artículo: Cardoso-Erlam, N., y Morales-Oliveros, E,E. (2017). Concepciones de tecnología en docentes universitarios de ciencias. Revista Científica, 30 (3), 195-206. Doi: https://doi.org/10.14483/23448350.12277

\section{Resumen}

A continuación, se presenta un resultado parcial de la investigación "Concepciones de ciencia, tecnología y las relaciones CTS en profesores universitarios y de formación media, y en textos escolares de ciencias". Enseguida se aborda la pregunta relacionada con las concepciones que sobre la naturaleza de la tecnología expresan los profesores universitarios de la Facultad de Ciencias de la Universidad del Tolima. La información se recogió mediante entrevista focal y el análisis de la información se realizó mediante análisis de contenido. Se encuentra que los profesores expresan diversas concepciones sobre la naturaleza de la tecnología, algunas más desactualizadas que otras. Solo un docente expresa una visión adecuada y actualizada.

Palabras clave: enseñanza científica superior, enseñanza de las ciencias, epistemología de la tecnología, naturaleza de la tecnología, profesores universitarios.

\begin{abstract}
Next, it's a partial result of the research investigation "Conceptions of sciences, technology and CTS relations with training academic, secondary teachers and sciences textbooks."Then the question about teachers' conceptions of the nature of technology is that teachers of the University of Tolima expressed is discussed. Information was collected through interviews and focus information analysis was performed using content analysis. It's found that teachers have different conceptions about the nature of technology some more outdated than others.
\end{abstract}

Keywords: Epistemology of science, science education. higher science education.

\section{Resumo}

Abaixo apresenta um resultado parcial de "concepções de ciência, tecnologia e pesquisa as relações CTS em acadêmicos e treinamento de mídia e em livros didáticos de ciência". Em seguida, aborda a 
questão das concepções sobre a natureza dos professores da Universidade expressas a tecnologia da faculdade de Ciências da Universidade de Tolima. A informação foi recolhida através de entrevista focal e análise de informações foi realizada através da análise de conteúdo. É que os professores expressaram diferentes concepções sobre a natureza da tecnologia, mais desatualizada do que outros. Um professor que expressa uma visão actualizada.

Palavras-chaves: epistemologia da tecnología, enseñanza de las ciencias, ensino superior científico.

\section{Introducción}

\section{Comprendiendo la naturaleza de la tecnología}

No existe consenso sobre el período de constitución de la tecnología como campo disciplinar, no obstante, podríamos ubicarla en el transcurso del siglo XIX con el desarrollo del capitalismo y la era industrial y hasta el final de la segunda guerra mundial. En el siglo XIX se dio la transformación de las labores artesanales o técnicas manuales hacia el uso de las herramientas y de aparatos-herramientas para facilitar la producción en masa y el comercio propio del nuevo sistema de producción. Esta forma de intercambio de bienes, especialmente de la industria textil y de la agricultura, exigió la investigación, el diseño y producción ya no de herramientas sino de equipos y sistemas sofisticados que requirieron de grandes plantas o fábricas. Los estudios de eficiencia de dichos equipos implementaron comparaciones de resistencia, duración, disponibilidad y costo de materiales eléctricos y electrónicos. La técnica, entonces, dio un paso importante hacia la tecnología por cuanto que, además de la determinación de la eficiencia, se hizo importante producir discurso específico, diseños e innovación que da inicio a la reflexión sobre las implicaciones sociales, éticas y ambientales.

El siglo XX fue el principal testigo del avance de la industria textil, farmacéutica y del transporte. La farmacéutica de botica y de los emplastos caseros se transformó en gran industria de la salud proveniente de los laboratorios. Para ello, la química aportaba sus investigaciones en función del control del dolor y la enfermedad. La industria floreciente del confort puso al alcance nuevos aparatos electrodomésticos para preservar y procesar alimentos; la agroindustria aportó valor agregado mediante el procesamiento de todo tipo de productos agrícolas para su comercialización. En esta implementación de lo moderno la mujer se distanció de las actividades de hogar, redujo las jornadas domésticas pasando a constituirse en obrera, oficinista y profesional. El nuevo vivir se relacionó con la posibilidad de escuchar la voz a distancia mediante la radio, el teléfono y la televisión, con lo cual las telecomunicaciones inician su carrera espléndida de desarrollo. Así mismo, el transporte redujo sustancialmente los tiempos de desplazamiento gracias a la ingeniería civil. De esta manera, los grandes aportes de la técnica en la vida cotidiana hacen "ver la ciencia como una máquina de solucionar problemas tecnológicos (Zimman, 1980).

La ingeniería de petróleo explora, explota y aporta a la producción de todo tipo de subproductos que supuestamente facilitan la vida. Entre tanto, la Guerra fría hizo también su trabajo investigativo y tecnológico; el poder se sofisticó para dominar la aeronáutica y la investigación química se implementó para superar la guerra cuerpo a cuerpo. La barbarie se masificó.

En más recientes años, la eugenesia irrumpe con sus modelos y experiencias, pero la bioética alerta de su posible mal uso. Entretanto, la nanotecnología se anuncia como la segunda gran revolución industrial para el siglo XXI, dado su campo de desarrollo que permitirá el ahorro significativo de energía y la reducción de peso y tamaño de los aparatos electrónicos. Así, las ingenierías, el diseño industrial y la salud serán las grandes transformadas. Actualmente, los microchips, la nanotecnología, la ingeniería genética y la física cuántica son expresiones que cada vez aportan desde lo no visible por el ojo humano al conocimiento de la 
realidad para la reducción del sufrimiento y al incremento del bienestar social.

Así, a partir del siglo XX el concepto de técnica transmutó en tecnología en la medida que sus procesos requirieron de mayor número de transformaciones, de mayor personal especializado, así como el ingreso de los aparatos a la vida familiar con la consecuente masificación. Estas transformaciones generaron confusión en cuanto a su definición, pues la expresión tecnología quedó asociada con los artefactos, la electrónica y la ciencia, por lo cual surgió la reflexión académica (Cupani, A. 2006).

Ahora se reconoce que técnica y tecnología son expresiones que aluden a aspectos apenas similares; mientras la técnica produce aparatos o herramientas, requiére de representaciones y la transformación de materiales para facilitar el trabajo en función de la eficacia, la tecnología alude al saber sobre lo técnico, esto es, que conlleva un discurso, una epistemología.

La tecnología como saber produce conocimiento sobre los factores representacionales y valorativos, esto es, analiza la esencia en cuanto a materiales, sus relaciones y posibles beneficios en función de la cultura. Por tanto, se acepta como campo objeto de investigación, líneas y problemas. Llegar allí obliga la acción de la epistemología y la axiología mediante preguntas sobre el qué, el para qué y el cómo que permitan superar la producción de saber técnico sin sentido y descontextualizado de la sociedad.

Para intentar su entendimiento, así inició el vencimiento de su demonización, esto es, que se fundó como un proceso de discusión ideológica y ética. Precisamente, de las concepciones epistemológicas que posean los profesores sobre tecnología se deriva en alto grado la actitud de los estudiantes hacia los estudios de tecnociencia.

Aunque la distinción entre ciencia y tecnología es difusa y vaga (Zimman, 1980), se entiende por tecnología una unidad junto con la ciencia y la ingeniería, que conforman una síntesis dialéctica pero que se diferencian y complementan. Según la Academia Norteamericana de Tecnología (2010):

La tecnología es un producto de la ingeniería y de la ciencia como parte de estudio del mundo natural. La ciencia tiene dos partes: un cuerpo de conocimientos que ha acumulado con el tiempo y un proceso de conocimiento-investigación científica-que genera sobre el mundo natural. La Ingeniería, también consta de un cuerpo de conocimientos-en este caso de los conocimientos en el diseño y creación de productos hechos por el hombre-y un proceso para resolver problemas. Mientras la ciencia tiene como objetivo entender el "por qué" y "cómo" de la naturaleza, la ingeniería busca dar forma al mundo natural para satisfacer las necesidades y deseos humanos. La ingeniería, por lo tanto, podría ser Ilamada "diseño bajo presión" por las leyes de la naturaleza como una serie de factores limitantes y que los ingenieros deben tener en cuenta. Otras restricciones incluyen el costo, la confiabilidad, seguridad, impacto ambiental, la facilidad de uso, los recursos humanos materiales, la fabricación, las regulaciones gubernamentales, e incluso los asuntos políticos. En resumen, la tecnología pasa necesariamente por la ciencia y la ingeniería.

Por la complejidad señalada resulta importante determinar algunos componentes que permitan configurar la naturaleza de la técnica, las implicaciones y relaciones con la sociedad, caracterización que se realiza a partir de los aportes que sobre la ciencia hace Adúriz-Bravo (2001) y que, ahora adaptados, permiten proponer una aproximación a su naturaleza. Estas acciones estructurantes son: la tecnología como acción discursiva, la tecnología como acción social cultural, la tecnología como acción utilitaria y la tecnología como acción sujeta a cuestionamiento. Se denominan acciones por cuanto que, como lo planteara Aristóteles, hacen parte de los procesos de perfección humana (Cáceres, 2000). 


\section{La tecnología como acción discursiva y la técnica como acción práctica}

La técnica se reconoce como pericia, habilidad, procedimientos y recursos. Es decir, se asume como artificio para transformar algo relativo al medio para alcanzar una meta o para reducir el tiempo que se requeriría para su logro. Es la eficiencia en el uso de objetos o de pasos para superar circunstancias difíciles o con alto gasto energético. Así, la historia de la sociedad humana, desde el Homo sapiens, ha recurrido a la técnica para adaptar el medio a través del simbolismo y la representación al punto que existen un sinnúmero de acciones técnicas que están incorporadas a la vida y que no requerimos razonarlas o cuestionarlas para aceptarlas como válidas o al menos útiles; son parte de los aprendizajes para adaptarnos y vivir en sociedad con cierto grado de solvencia.

Como señala Agassi (1997), el término técnica en inglés es casi inusual, en cambio en español es más corriente al punto que podría distinguirse de tecnología para señalarla como asunto en la cual lo mecánico es primordial. Esto es que es repetitivo en su esencia. Lo técnico, al menos sustancialmente, no ha requerido de modelos matemáticos o fórmulas previas; es el resultado, especialmente, de ensayo y error, del perfeccionamiento de una práctica que generación tras generación se ha transformado, cualificado y estabilizado. Por su parte, lo tecnológico requiere del pensamiento analógico y visual sin descartar la abstracción (Cupani, 2006) y se fundamenta en la eficiencia; es expresión que posee mayor uso en la vida cotidiana occidental y de manera especial en los países de mayor desarrollo industrial.

La expresión tecnología por definición se compone de tecné y logos. Es decir, dilucidar y comprender, alude al estudio de la técnica y en consecuencia, su teoría. "Es el conjunto de conocimiento organizado sobre las técnicas" (Zimman, 1980). Este es el salto cualitativo que se acompaña de la epistemología como intento de dar razón de las cosas: ¿qué es?, ¿para qué?, ¿cómo funciona? ¿qué connotaciones éticas implica? Esta comprensión, junto con la ciencia de punta, es una transformación de la representación de la técnica y permite diferenciar el simple utilitarismo de la adecuada utilidad; entonces, la cosa en sí pasa a ser cosa pensada. Producto de este ejercicio de la razón, surgen diversos pensamientos, interpretaciones, discursos y teorías distintas que nos permite construir una epistemología de la técnica, o del arte (según el sentido griego antiguo) esto es de la tecnología (Agassi, 1997).

Así entonces, tenemos que toda tecnología incluye una técnica, pero no toda técnica incluye una tecnología; como afirma Zimman (1980) "no toda la información tecnológica se basa en la ciencia y no toda la ciencia se deriva de la tecnología". La validez de la técnica no se obtiene como la de la ciencia, por su plausibilidad inherente o por la comunicabilidad y aceptación académica sino por su éxito práctico (Simpan, 1980) por lo que se convierten en objeto de análisis axiológico.

Esta complejidad es explicada por Agassi (1997): "como tema interrelacionado de conocimientos, artefactos, destrezas y habilidades, recursos naturales, estimaciones económicas, valores y acuerdos sociales, preferencias culturales y estéticas, esto es un entramado sociotécnico".

Allí se reconoce que, tanto la ciencia como la tecnología se mueven políticamente en dos niveles: el internacional, propio del mundo diplomático, y el nacional con sus intereses políticos y socioeconómicos gubernamentales (Aikenhead, 1994), en consecuencia, sin neutralidad. Entramado que tiene de fondo los sistemas educativos; como señalan Vázquez y Manassero (2008) y Cardoso y Morales (2009), la formación mediante prácticas investigativas en las Facultades de Ciencias y Tecnología es insuficiente para enseñar CTS- NDCyT.

La visión descontextualizada de la ciencia se ha trasladado a la de tecnología. Como señala Aikenhaed (1994), los científicos desde el siglo XX definieron la tecnología como ciencia aplicada, concepción que sigue hoy, erróneamente, en el imaginario social pues aún se considera que esta 
es resultado directo de la ciencia y que posee menor estatus (Caphapuz et al., 2005). Lo cierto es que la relación de la tecnología con la ciencia puede ser unívoca o biunívoca. Precisamente, el movimiento denominado alfabetización científica y tecnológica para todos y todas incluye en sus debates la sociología de la ciencia y de la tecnología, atendiendo el estudio de los contextos sociales y culturales.

La producción de conocimiento no es una actividad aislada, es una construcción del hombre en cuanto ser histórico, adscrito a una cultura en particular. Esta cultura genera acciones y reacciones políticas y manifestaciones de segmentos de sociedad (religiosa, civil, profesional) que la transforma o retrasa. Por su parte, los científicos como comunidad realizan sus cuestionamientos internos sobre la ciencia y la defienden en un contexto que estimula o por el contrario es adversa a las innovaciones. Esto es que, las corrientes socioculturales permean y filtran dichas concepciones y evolución. La antigua ecuación de a mayor ciencia = mayor tecnología = mayor riqueza= mayor bienestar, representa la forma lineal y errónea de entender la relación CTS (Chrispino, 2008). Esta es una concepción centrada en una particular percepción de progreso como sinónimo de dinero con menor esfuerzo y para un supuesto bienestar imparable sin el reconocimiento de las transformaciones negativas del entorno. La comprensión de los alcances y limitaciones de la tecnología en la sociedad genera la necesidad de repensarla, esto es reconocer su verdad, su ética y su estética, razón por la cual se ha consolidado la epistemología de la ciencia y de la tecnología, "más que un problema de uso de nuevas tecnologías es un problema cognitivo" (Colciencias, 2008).

La tecnología como acción utilitaria, en donde el sentido de utilidad está referido al saber producido mediante el diseño, la modelización y el discurso específico que sustenta la técnica como práctica. En este sentido, se alude a los aspectos epistémicos que dan cuenta de la historia de la técnica, sus aportes, su construcción y razón de ser. Además del estudio de las implicaciones por el real o aparente confort derivado, la rapidez o facilidad y efectos de mediano y largo plazo en la vida cotidiana y la cultura. Entonces, cuando nos referimos a tecnología no lo hacemos sobre los resultados de la técnica como son los artefactos y aparatos per sé, fuera de contexto. En el debate histórico ha estado la visión de tecnología como sinónimo de lo artificial. Por tanto, a veces, contra la naturaleza, actualmente se asocia con el daño ambiental y su necesaria protección, pero sin el ingrediente moralista.

La tecnología como acción cuestionadora y cuestionante de toda obra humana genera alguna reacción social que la afecta positiva o negativamente. Sus cuestionamientos se debaten con creencias, juicios, prejuicios y principios de la ciencia que condicionan su incremento y uso. La técnica de punta lleva a pensar en la afectación de los valores y, a su vez, nos hace cuestionar sobre la idea de hombre como ser en permanente proceso de perfeccionamiento. Para Vega (2009) la tecnología ha sido considerada como una "subdisciplina de la filosofía, viene a ser como la hermana menor de la epistemología", de la filosofía, estatus surgido en la década de 1960. Así, la ética tecnológica, que se enmarca en la bioética, la ética medioambiental y la ética de la ciencia, se constituye en campo de estudio para determinar y sopesar el valor de lo utilitario y de las innovaciones. Una sociedad realmente actualizada y éticamente preparada para la toma de decisiones sobre CyT requiere tanto de una puesta en común orientada a la formación en este sentido (Aikenhead, 1997; Lederman, 1992) como a una afortunada adecuación curricular (Chirspino, 2008; Manassero y Vázquez, 2001).

En este escenario de balance y prospectiva, las academias en Estados Unidos han planteado estándares mínimos para un buen desempeño de los profesores de tecnociencias y uso de las TIC. Se espera que los profesores promuevan y apoyen el modelamiento, la creatividad e innovación para la solución de problemas y la exploración de temas 
del mundo real y la solución de problemas. De similar manera, el diseño y las pruebas propias de la innovación y la transferencia tecnológica requieren del uso óptimo de herramientas digitales para localizar, analizar, evaluar y utilizar recursos, tal como lo señalan los citados estándares. Como se infiere, el acercamiento a lo tecnológico es a partir de las TIC para lograr un adecuada aproximación a los procesos de desarrollo mental (ciencia) y de ciudadanía (responsabilidad de uso).

La Asociación Internacional de Educadores de Tecnología e Ingeniería (Iteea en inglés), produce, desde hace varios años, conocimiento y orientación para los profesores de ingeniería y de tecnologías al punto que se han establecido como factor de referencia para el conocimiento epistemológico, pedagógico y didáctico de la ingeniería y en general la tecnociencia. Así mismo, están influyendo en los currículos en los diversos niveles a través de libros que aportan a su desarrollo, conocimiento y discusión. Por su parte, la Internacional Society for Techology in Education (Iste) ha propuesto unos estándares para los estudiantes en el sentido de qué es lo que ellos deben saber y qué habilidades deben poseer para aprender efectivamente $y$ vivir productivamente en un mundo cada vez más digital y tecnologizado.

Este es un campo, por supuesto, obligado por el Ministerio de Educación Nacional, colombiano (1998), a través de los lineamientos curriculares en la formación media, sin embargo, los profesores no poseen formación específica para la discusión sobre tales concepciones. Se reconoce que ciencia y tecnología es un contrato social, pero que infortunadamente cuando se enseña ciencias este vínculo desaparece; nuestros cursos en la educación media y superior están centrados más en los contenidos de ciencias dejando relegados los asuntos sobre la tecnología. De aquí se desprende la hipótesis de trabajo para esta parte de la investigación: no hay conocimiento suficiente sobre las concepciones de los docentes de ciencias sobre la tecnología y su relación con la ciencia. En este sentido, el estudio indaga tal aspecto en profesores universitarios de Ibagué bajo la pregunta central ¿cuáles son las concepciones de tecnología que muestran los docentes de la Facultad de Ciencias de la Universidad del Tolima? ¿Son adecuadas o desactualizadas tales creencias?

\section{Método}

El objetivo era conocer qué pensaban y sabían sobre la naturaleza de la tecnología y su relación con la ciencia un grupo de profesores de planta de los departamentos de Física, Química y Biología de la Facultad de Ciencias Básicas de la Universidad del Tolima. Ello mediante un guion básico, con el cual se realizaron entrevistas focales que se grabaron en formato digital de audio y video, luego se transcribieron en el procesador de texto Word, con lo cual se procesó la información mediante el análisis de contenido (Bardín, 1996; Klipendorf, 1997). El informe se presenta mediante el apoyo de las frases textuales de las entrevistas y el análisis de sentido o metaanálisis referido a la emergencia de las concepciones epistemológicas de los docentes. Con lo anterior, se determina que la perspectiva investigativa es de carácter hermenéutico. Las entrevistas se realizaron en tres momentos, entre los meses de agosto y octubre de 2010, con duración aproximada de una hora y media.

\section{Resultados}

\section{Campos estructurantes de la tecnología según profesores universitarios}

\section{La técnica como acción práctica y la tecnología como acción discursiva}

Para los profesores universitarios de este estudio, su visión sobre la tecnología es reconocida como equivalente a ciencia aplicada, esto es como un consecuente de la ciencia. Así lo refiere un docente al preguntársele sobre dicha relación: "quisiera que me mencionaran una sola situación de ciencia aplicada sin que previamente haya existido ciencia 
básica, no la hay, no la hay. Entonces hay que entender que eso es como un matrimonio" (8.1).

Si bien es cierto que la ciencia es producción de conocimiento sin un fin práctico inmediato, no significa que lo excluya; no existe un vacío entre la ciencia básica y la ciencia aplicada quizá, el asunto sea de magnitud o cobertura de la utilidad. Sin embargo, el uso de la analogía (relación similar al matrimonio) deja en el vacío el argumento anterior. De hecho, es cierto que las orientaciones científicas están jalonadas por los rendimientos y aprovechamientos de la ingeniería para la aplicación y beneficio de la sociedad, pero también es cierto que algunos descubrimientos de las ciencias básicas han sido producto de hechos fortuitos e inesperados; los ejemplos en la historia de la ciencia abundan.

La tecnología directamente relacionada con el aporte a la innovación y al desarrollo se manifiesta de manera directa y estrecha en la ingeniería, son relaciones de doble vía intrínseca y prácticamente inseparable.

Según un profesor de física sobre el Gran Colisionador de Hadrones (LHC), señala que:

Se requiere utilizar unos imanes superconductores que exija a los ingenieros, uno les dice (a los ingenieros) mire necesito esto, ellos lo van a diseñar, entonces esa es la tecnología... se van a conseguir unas temperaturas muy altas, entonces se requiere un sistema de enfriamiento; ingenieros, necesitamos enfriar esto ¿cómo lo harían?, entonces se crean situaciones que son un desafío a la par de la ingeniería y así avanza la parte tecnológica, en este momento hay un reto muy grande, una aplicación inmediata a estos aceleradores de partículas... entonces la tecnología ¿qué es lo que hace? aplicar, ¿cierto? (8.1)

El primer número corresponde al departamento al cual está adscrito el docente. El segundo al profesor entrevistado. Al departamento de Química se le asignó el número 7, al de Física el 8 y al de Biología el 9. Por su parte, otros docentes expresan una visión entre contradictoria y errónea:
La tecnología se genera de la ciencia, en la medida en que le plantea retos para solucionar de una manera más efectiva una problemática o un problema en particular... uno piensa que la tecnología está y debe estar sujeta al desarrollo de la ciencia y ésta a su vez está generando tecnología. (9.4)

Sin embargo, también existe evidencia de una concepción diferente, contrapuesta y más actualizada, por cuanto que menciona tácitamente la relación biunívoca entre ciencia, técnica y tecnología por parte de algunos profesores. Veamos un ejemplo:

Hoy en día uno no pueda decir que primero es esto (ciencia) y que después lo otro (técnica), uno diría que ese avance tecnológico puede producir un conocimiento que después va a reforzar, puede ser que los conocimientos tecnológicos también den lugar a un conocimiento básico que después pueda ser revertido. (9.2)

Esta visión es confirmada por otro docente cuando explicita:

Bueno, yo difiero de la concepción que tiene el profesor 2 sobre tecnología. Yo considero que ciencia y tecnología son dos campos independientes, ¿sí?, pues el término tecnología, digamos, es el tratado de las técnicas; hemos hablado acá que ciencias es un conjunto de conocimientos, o sea la ciencia es algo teórico, ¿cierto? Como conjunto de conocimientos es teórico, porque son conocimientos obtenidos de la naturaleza pero que posteriormente se plantean dentro del campo teórico. (9.1)

Hemos planteado que la tecnología es el estudio de los procesos técnicos y su desarrollo que produce un saber de tipo tecnocientífico. Pero ¿qué dicen los profesores al respecto? Veamos: "Hay mucho trabajo de ingeniería, entonces ahí se ve cómo ese matrimonio entre tres, entre ciencia básica, ciencia aplicada y tecnología, digamos funciona y hay un momento donde se confunde todo" (8.1). 
A partir de lo anterior, no es claro si es una noción de complejidad o de confusión. Además, la analogía del matrimonio, que regularmente es entre dos, acá se explica cómo relación de tres, con lo que resulta inadecuada la comparación. Un aspecto importante es que las ideas de ciencia y tecnología resultan a veces asociadas con la del sujeto de la acción. Se habla de ciencia y de tecnología como si siempre fueran realizadas por sujetos distintos, el científico quien solo hace ciencia y el técnico solo tecnología. Quien hace ciencia probablemente no intervenga en la construcción de los aparatos o sistemas técnicos, aunque su diseño y conocimiento de funcionamiento no le es desconocido, es más, le es pertinente y necesario.

De otra parte, quien hace tecnología no es solo un técnico, es, sin duda, también científico al diseñar, construir, probar y evaluar los proyectos. Entonces, como afirma un profesor que excluye la producción de saber derivado de la tecnología al referirse a la ciencia: "cuando hablamos de ciencia hablamos de generación de conocimiento, de búsqueda de respuestas a preguntas que nos resultan del entorno" (7.7). Separación que resulta inadecuada, sobre todo cuando entendemos que precisamente la tecnociencia se produce en grandes equipos interdisciplinares, donde todos están aportando ideas, modelos y diseños, discutiendo sus alcances y su relación costo beneficio social. Por este motivo, afirmaciones como que cuando hay un problema tecnológico es asunto exclusivo de la tecnología no resultan adecuadas: "hay que Ilevarle esa inquietud a la ciencia para ver que pasó" (9.4). Esto es, entender la tecnología como mero asunto práctico y la ciencia como a quien le corresponde atender los problemas derivados de lo tecnológico. Es algo así, la ciencia (el científico) piensa y resuelve y la tecnología (el tecnólogo) aplica sin pensar.

\section{La tecnología como acción sociocultural}

El estudio de las relaciones CTyS se han constituido en asunto de relevancia para una adecuada alfabetización científica, movimiento mundial que busca incrementar la discusión sobre los beneficios y consecuencias negativas del desarrollo. Por ello, es importante que los currículos incorporen estos temas, para lo cual es importante que los profesores posean una adecuada expectativa y referencia al respecto. Entonces, veamos qué plantean nuestros docentes entrevistados. Un profesor establece la necesidad de que científicos y tecnólogos se constituyan en catalizadores de las implicaciones sociopolíticas, así lo expresa un docente:

La ciencia y la tecnología no son panacea, sí son como toda herramienta, que pueden tener ventajas y desventajas, entonces lo que pasa es que tiene que haber una evaluación por parte de grupos de la misma comunidad científica del país. (9.1)

Afirmación que permite inferir la exclusión, o al menos, de no tener en cuenta a la sociedad para la opción de discutir y aportar a la toma de decisiones democráticas tal como lo explica el movimiento alfabetización científica para todos y todas.

La comprensión de las expresiones técnica y tecnología es parcialmente valedera si observamos las siguientes manifestaciones: "Técnica es simplemente una herramienta. Las técnicas han surgido históricamente como inventos, o herramientas que el hombre utiliza para manejar la naturaleza; la técnica es una herramienta que el hombre interpone entre sí y el objeto que estudia" (9.1).

Técnica no es simplemente una herramienta, la herramienta es un instrumento que facilita el trabajo. Sin embargo, al agregarle la expresión invento anula lo anteriormente afirmado, pues la inventiva no es herramienta, es pensamiento analógico, requiere del diseño y superación de una dificultad para la cual no hay aún respuesta. La técnica es resultado de los avances científicos y las adecuaciones tecnológicas, que en su punto más alto han derivado en la denominada tecnociencia. Es decir, existe, de una parte, la técnica primitiva, rupestre o elemental y de otra parte, la técnica avanzada, de punta que recurre a lo digital, los microchips, 
lo virtual, electromagnético etc. "Para que la tecnología avance se requiere primero fortalecer el conocimiento; la ciencia tiene que generar ese conocimiento a través de todas las posibilidades de herramientas" (9.2).

Esta afirmación va en contravía del primer planteamiento, pues mientras el primer docente pretende aclarar el límite conceptual de las herramientas, el segundo asume que para que exista tecnología está de base la ciencia, es consecuencia. Según se deriva, la tecnología no produce conocimiento sobre sí misma. Para otra profesora la técnica es como

Un formato, como una herramienta, pero no puede ser tan separada porque hay procesos de la tecnología que llevan a mirar inquietudes y los retos al mismo científico que está apoyando esa tecnología. Si la vemos sólo como una herramienta que se generó por un conocimiento para que la apliquen, tampoco es así. (9.1)

De nuevo se percibe la supuesta dependencia unilateral de la tecnología a partir de la ciencia.

Sin embargo, también existen segmentos de entrevista con versiones más adecuadas como la que denota que la tecnología incluye procesos y diseños particulares a su hacer lo que es evidente en la afirmación de la misma docente (9.1) cuando afirma "la tecnología no simplemente está sujeta a la innovación de aparatos sino también a los procedimientos y protocolos".

Entre tanto, sobre el carácter de beneficio social que debe tener la ciencia y la tecnología, esto es, las relaciones externas, un docente hace una intervención haciendo notar ciertos avances, al menos en la región:

Actualmente los comités departamentales de ciencia y tecnología, o lo que se hace con la alianza, universidad-empresa-estado está empezando a cambiar. ¿Qué pasaba antes? (Los resultados) terminaban siendo para los investigadores... no terminaba en una innovación que revirtiera en la comunidad y el ejemplo que les digo ocurría con comunidades campesinas. (7.6)

Los comités departamentales de ciencia y tecnología efectivamente buscan atraer conocimiento producido en las universidades colombianas para acercarlo a las comunidades en función de mejor productividad. Esto es un ejemplo sobre cómo la sociedad organiza y exige pertinencia y eficiencia en tales procesos, el asunto es que son emprendimientos lentos y de relativa dificultad para su implementación. En este sentido, las relaciones externas CyT son acciones vivas, se dinamizan como producto de las tensiones políticas y comunitarias.

\section{La tecnología como acción utilitaria}

La tecnología generalmente está asociada con la innovación, la función de utilidad y la eficiencia de ciertos aparatos de uso cotidiano. Esta relación es comúnmente desligada de la ciencia, esto es, que la tecnología se encarga de producir artefactos y la ciencia se encarga del conocimiento, asuntos que se también se vislumbran en apartados anteriores.

Son diversas las concepciones respecto a la implementación de la tecnología. Al respecto, un profesor anota que en la minería en Colombia no se ha implementado de manera amplia la tecnología, dado que:

Los sistemas más rupestres en minería están en Boyacá, por la zona de norte de Antioquia; Amagá y Amalfi, son zonas donde los entornos culturales están aislados con respecto a lo que podía ser el conocimiento y el manejo de un sistema tecnológico para hacer un uso benéfico. (7.6)

El docente en su declaración hace ver la estrecha relación que debe existir entre la técnica avanzada y la vida productiva pero que en nuestro país está aún pendiente.

Otro docente plantea, de nuevo, la relación unívoca entre tecnología y ciencia de manera 
casuística cuando explica que los estudios sobre física cuántica se generaron mucho tiempo antes que las aplicaciones tecnológicas dado que no se conocían las aplicaciones derivadas de dichos hallazgos. Cuando hacia 1920 los investigadores iniciaban sus avances no esperaban aportar a la dimensionalidad para producir aparatos tales como la fibra óptica (9.2).

En la ciencia y la tecnología, aunque las estrategias y protocolos pueden diferir son separables, al punto que existe un efecto espiral por el cual si un país es avanzado en desarrollos científicos igualmente lo es en tecnología. Al respecto, un docente es claro al señalar el error usual en gran parte de la sociedad al considerar que como otros países ya poseen ciertos adelantos bastaría con comprarlos e importarlos pues esto sería más práctico y económico, concepción que resulta como "una mirada muy pobre" (9.2). Estos desarrollos necesitan de grandes presupuestos para lo cual se presenta la alternativa de adquirir patentes, con la consecuente dependencia, o invertir en la producción de dicha ciencia y técnica. En este caso el docente tiene en cuenta que en las decisiones de tecnociencia deben intervenir diversos actores de la sociedad.

En resumen, el docente codificado como 9.1 es el único que denota una concepción más acorde con los planteamientos epistemológicos actuales de la tecnología. Explica adecuadamente la relación de interdependencia entre ciencia y tecnología subrayando que esta no depende de la primera. Por el contrario, que en cuanto al diseño y producción existen procederes diferentes en la técnica que generan conocimiento y alimentan a la ciencia y a sí misma. Por su parte, la mayoría de docentes muestran y dejan entrever una concepción lineal sobre ciencia y tecnología pues la refieren como de dependencia unilateral y aún queda implícita la idea que la tecnología hace mención a los artefactos que facilitan hacer ciencia y a aquellos que ingresan a la vida cotidiana para mayor confort. Una frase que puede encerrar esta visión de varias décadas atrás es: "Nosotros, que nacimos mucho tiempo después de la mecánica cuántica, estamos gozando de las aplicaciones tecnológicas de la misma, en aquella época era imposible saber para dónde se iba en aplicaciones tecnológicas" (8.1).

Reflexión que sí es referida por otro docente cuando alude a la importancia de la discusión filosófica para conocer el real beneficio que puedan aportar los artefactos diseñados para la comodidad o si se otra forma de esclavitud de la sociedad (9.1). Es usual la creencia que la tecnociencia va dirigida a resolver los problemas de la sociedad (9.3), asunto que reclama análisis sociopolítico para establecer si son necesidades reales o creadas en un entorno donde millones de personas mueren por falta de asistencia alimentaria y médica, por ejemplo.

Un docente expresa acertadamente que la tecnología es la ciencia de las técnicas, de los aparatos, de los procedimientos, por lo que debe haber una epistemología. Esta ciencia de la técnica se relaciona con que: "usa parte del conocimiento pero también cuando diseña cosas, puede haber novedades, pueden generar inquietudes para volver a mirarlo desde un punto de vista científico, produciendo nuevos mejoramientos, adaptaciones, etc.". (9.3).

\section{Conclusiones}

En los docentes coexisten diversas interpretaciones sobre la relación de dependencia o interdependencia entre la ciencia y la tecnología. Varios docentes, al realizar la explicación sobre la naturaleza de la tecnología, no la separan de la ciencia, pero, a su vez, erróneamente la consideran como dependiente y herramienta al servicio de la ciencia. De los docentes entrevistados solo uno muestra un adecuado y actualizado concepto sobre la tecnología al caracterizar su naturaleza en el justo nivel de complejidad e interdependencia. Es decir, solo un profesor reconoce que la tecnología es el estudio de la técnica y que interactúa en doble sentido con la ciencia, que incluye un discurso y que sus aplicaciones están mediadas por factores sociopolíticos. 
Así entonces, las afirmaciones de la mayoría de profesores corresponden a las concepciones y creencias bastante desactualizadas. Con respecto al departamento en el que laboran, los docentes de Biología y Física expresan una concepción menos errónea que los de química, aunque en cada uno de estos existen algunas ideas adecuadas. La tecnología asociada a las herramientas o aparatos únicamente se hace evidente en la mayoría de los profesores. Solo un profesor señala y explica la diferencia conceptual entre ciencia, técnica y tecnología de manera que la visión predominante son las múltiples formas de relacionar estas tres acciones.

Al analizar las diversas ideas que expresan los docentes universitarios, algo interesante para analizar es cómo es el uso de analogías para la relación entre ciencia y tecnología. Se utilizaron expresiones tales como: hija, hermana, matrimonio e inclusive como disciplinas que van de la mano. Son referencias que de alguna manera suavizan los conceptos, utilizadas como una manera cotidiana de entender esa relación, sin embargo, resultan inadecuadas.

Es de anotar también que al referirse a la naturaleza de la tecnología ningún docente la determinó como producto humano, cultural e históricamente determinada. Pareciera que dependiera de la ciencia y que esta tampoco tuviera como base la sociedad. En otras palabras, su procedencia derivada de la construcción humana y de unas necesidades reales y creadas por la sociedad es escasa. Con respecto a los tres tipos de acciones propuestas (discursiva, socio cultural y utilitaria) que implica la naturaleza de la ciencia no se esperaba que fueran mencionadas de manera explícita, pero sí que de manera implícita o tangencial se hiciese alusión a tales factores que la determinan.

En resumen, el profesorado no muestra un adecuado nivel de conocimiento sobre la tecnología como para esperar que su enseñanza sea actualizada y eficiente. Bajo esta premisa difícilmente se podría esperar que el sentido sociocultural y de utilidad de lo tecnológica no sea tergiversado y que los estudiantes, en consecuencia, no se están beneficiando adecuadamente con tales aportes. Así, es indispensable establecer programas de formación docente permanente con el objetivo central de modificar dichas concepciones que aporten marcando diferencia y apunten al propósito internacional del movimiento alfabetización científica y tecnológica para todos y todas.

\section{Agradecimientos}

El proyecto investigación "Concepciones de ciencia, tecnología y las relaciones CTS en profesores universitarios y de formación media, y en textos escolares de ciencias" es financiado por el Fondo del Comité Central de Investigaciones de la Universidad del Tolima. Código: 40108.

\section{Referencias}

Aduriz-Bravo, A. (2001). Integración de la epistemología en la formación del profesorado de ciencias (tesis de doctorado). Programa de Doctorado en Didáctica de las Ciencias Experimentales, Universidad Autónoma de Barcelona, Barcelona, España. Recuperado de: http:// www.tdx.cesca.es/TDCat-1209102-142933.

Agazzi, E. (1997). El impacto epistemológico de la tecnología. Italia: Universidad de Génova. Recuperado de: www.argumentos.es/numero1/ Agassi.htm

Aikenhead, G. S. (1994). The social contract of science: Implications for teaching science. En: J. Solomon y G. S. Aikenhead (eds.), STS Education: International Perspectives onReform (pp. 11-20). Nueva York: Teachers College Press.

Aikenhead, G. S. (1997). Exploring Ideologies: STS and HPS. Presentado en HPS, STS and thegoals of schoolscience en la conferencia Historia y Filosofía de la ciencia y enseñanza de la ciencia. Calgary, Canadá, June 21-24. Revisado en marzo 2010: http://www.usask.ca/education/people/aikenhead/explideo.htm 
Bardín, L. (1996). Análisis de contenido. Madrid: Akal Editores.

Cáceres, E., Gil, M. (2000). La techné y la técnica moderna: una aproximación teórica. Cuaderno de Relaciones laborales. 16, 99-132.

Caphapuz, A. et al. (2005). À necessáriarenovação do ensino das ciências. São Paulo: Cortez Editores.

Cardoso, N y Morales, E. (2009). Actitudes hacia la naturaleza de las ciencias $(\mathrm{NdC})$ en profesores de ciencias y matemáticas (CyM) y profesores de humanidades y sociales (HyS). Avance de un estudio comparativo. Enseñanza de las ciencias revista de investigación y experiencias didácticas, número extraordinario, 2390-2394.

Chrispino, A. O. (2008). Enfoque CTS- Ciencia, Tecnologia e socieda de e seis impactos no ensino. Tecnlogia y Cultura, 10(13), 7-17.

Departamento Administrativo de Ciencia, Tecnología e Innovación (Colciencias). (2008). Colombia, construye y siembra futuro. Política nacional de fomento a la investigación y la innovación. Bogotá: Colciencias.

Klipenndorff, K. (1997). Metodología de análisis de contenido. Teoría y práctica. Traducción de Leandro Wolf. Barcelona: Editorial Paídos.

Lederman, N. (1992). Student's and teachers conception of nature of science: a review of the research. Journal of research in science teaching, 29(4), 331-359.
Manassero, M.A. y Vázquez, A. (2002). Las concepciones de estudiantes y profesores de ciencia, tecnología y su relación: consecuencias para la educación. Revista de Ciencias de la Educación, 191, 315-343.

Manassero, M.A., Vázquez, A. y Acevedo, J.A. (2001). Avaluaciódels temes de ciència, tecnología i societat. Palma de Mallorca, España: Conselleriad Educació i Cultura del Govern de les Illes Ballears.

Ministerio de Educación Nacional (MEN). (1998). Lineamientos curriculares. Bogotá: MEN.

National Academy of Inginnering. (2010). The Nature of technology. Recuperado de:

Vázquez, A. y Manassero, M. (2007). La relevancia de la educación científica. Palma de Mallorca, España: Universitatlles Balears.

Vázquez, A., Acevedo, J. A., Manassero, M. A. y Acevedo, P. (2001). Cuatro paradigmas básicos sobre la naturaleza de la ciencia. Argumentos de Razón Técnica, 4, 135- 176.

Vázquez, A., Acevedo, J. A., Manassero, M. A. y Acevedo, P. (2007). Consensos sobre la naturaleza de la ciencia: la ciencia y la tecnología en la sociedad. Educación Química, 18(1), 38-55.

Vega, J. (2009). Estado de la cuestión, filosofía de la tecnología. Theoria, 66, 323-341.

Zimman, J. (1980). Enseñanza y aprendizaje sobre ciencia y sociedad. México: Fondo de Cultura Económica. 